Accepted for publication in "Clinical Psychological Science"

Note: This is an uncorrected version of an author's manuscript accepted for publication. Copyediting, typesetting, and review of the resulting proofs will be undertaken on this manuscript before final publication. During production and pre-press, errors may be discovered that could affect the content.

\title{
Rest-related dynamics of risk and protective factors for depression:
}

\author{
A behavioral study \\ Igor Marchetti, Ernst H.W. Koster, \& Rudi De Raedt \\ Department of Experimental Clinical and Health Psychology \\ Ghent University, Belgium
}

Brief Empirical Article

Word count: 4997

Corresponding Author: Igor Marchetti, Ghent University, Department of ExperimentalClinical and Health Psychology, E-mail: Igor.Marchetti@Ugent.be 
Running head: REST, SELF-FOCUS AND NEGATIVE MOOD

Abstract

Resting state provides an important condition to study depressogenic cognition as neuropsychological studies have associated rest with heightened self-related processing. We examined outside the fMRI scanner relationships between rest-related internal focus, cognitive reactivity (vs. mindfulness), rumination, and negative mood in an undergraduate sample $(\mathrm{N}=80)$. We tested a theory-based (Marchetti et al., 2012) effect by which, in presence of cognitive risk (vs. protective) factors, internal focus lowers mood via ruminative self-focus. Such effect was detected in individuals with high cognitive reactivity, whereas brooding only showed an incremental effect. On the contrary, this dynamic was not significant in individuals with low cognitive reactivity, despite the level of brooding, or high mindfulness. These results provide an important window on risk for depressogenic thought during resting state.

Keywords: resting state, attention, cognitive reactivity, rumination, mindfulness, mood 
Running head: REST, SELF-FOCUS AND NEGATIVE MOOD

"A mind quite vacant is a mind distress' $d$ "

- William Cowper (1731 - 1800)

\section{Introduction}

Resting state is defined as a state of relaxed wakefulness during which one is not actively engaged in any task, but one's mind is free to wander off. Despite such apparent idleness, our mind is constantly active and tends to gravitate towards internally-originated scenarios (e.g. daydreaming, involuntary autobiographical memories, etc.). In recent years, neurobiological research has confirmed such notion, highlighting that several large-scale neural networks intensively perpetuate their activation during rest (Broyd et al., 2009). Neuroimaging studies indicate that resting state (i.e. people quietly lie in the scanner with eyes closed) is associated with a neural network which shows a tight temporally synchronized co-activation of brain areas (Fox et al., 2005), the so-called Default Mode Network (DMN; Raichle et al., 2001). The DMN consists of two highly linked sub-networks showing anticorrelated activation, namely the Task Negative Network (TN) and the Task Positive Network (TP) (Broyd et al., 2009). TN is comprised of brain areas that are typically active during rest, such as medioprefrontal cortex and posterior cingulate (Raichle et al., 2001).

Interestingly, several psychological functions have been proposed to be embedded in the TN, such as autobiographical recalling, future prospection, self-referential thinking, and associative processing (Buckner et al., 2008). Broadly speaking, TN is thought to subserve internal focus, during which internally-generated information dominates (Fox et al., 2005) and self-related processing occurs (Northoff, 2012). TP consists of brain areas (e.g. dorsolateral prefrontal cortex and inferior parietal cortex) that are particularly active during non-specific task engagement, such as attention demanding tasks (Corbetta \& Shulman, 2002). For these reasons, TP is the putative neural substrate of external attentional focus (Fox 
et al., 2005). In sum, the DMN is a resting state network comprising highly anticorrelated oscillations between internal and external focus. Given the pivotal role of the DMN in both self-related internal focus and task-related engagement, DMN aberrations could play a major role in psychopathology (Broyd et al., 2009). For instance, increased TN activation during rest is found in depression and schizophrenia (for a review see, Whitfield-Gabrieli \& Ford, 2012). In depression, there is emerging data on the association between TN and maladaptive self-related processing, such as rumination (Berman et al., 2011). However, to date resting state studies have mostly examined isolated risk factors. Recently a comprehensive theoretical framework has been proposed relating various cognitive risk factors for depression to the DMN (Marchetti, Koster, Sonuga-Barke, \& De Raedt, 2012). The authors proposed that impaired attentional control, rumination, and cognitive reactivity are outcomes of DMN dysfunctions. The present study examines resting state in relation to rumination and cognitive reactivity at the behavioral level ${ }^{1}$.

Rumination has been defined as "repetitively focusing on the fact that one is depressed; on one's symptoms of depression; and on the causes, meanings, and consequences of depressive symptoms" (Nolen-Hoeksema, 1991, p.569). The deleterious impact of rumination may not derive from attention to distress per se, but from internally-oriented attention that is negative, evaluative, and judgmental (Rude, Maestas, \& Neff, 2007). Indeed, recent literature shows rumination to have its neural roots in increased $\mathrm{TN}$ activation during rest (Hamilton et al., 2011). Mainly, brooding -the most maladaptive form of rumination (i.e. passively focusing on the presence and meaning of symptoms of distress; Treynor, Gonzalez, \& Nolen-Hoeksema, 2003)-, is related to TN during rest (Berman et al., 2011). On the contrary, reflection (i.e. the degree to which individuals engage in problem solving to improve mood), considered as the more adaptive form of rumination, is negatively associated with temporal dominance of TN over TP (Hamilton et al., 2011). In sum, Marchetti et al. (2012) 
Running head: REST, SELF-FOCUS AND NEGATIVE MOOD

proposed that during rest increased TN activity is specifically associated with higher levels of maladaptive rumination (i.e. brooding).

The second rest-related risk factor is cognitive reactivity, namely fluctuations in the activation of negative self-attitudes in response to stressors (Butler, Hokanson, \& Flynn, 1994). The ease by which negative self-related thinking is triggered is accounted for by the Differential Activation Hypothesis (DAH; Teasdale, 1988). In brief, the DAH postulates that depressive episodes strengthen associative links between negative self-schemata, negative mood, and negative thinking, so that even mild stressors can activate a negative cascade in self-attitudes, depressive thinking, and related behavioral outcomes (e.g., depressive withdrawal). However, cognitive reactivity has been recently conceptualized as a process which can be observed also in at-risk individuals who never suffered from clinical depression (Moulds et al., 2008). Crucial for our study, the construct of cognitive reactivity assumes the existence of negative self-schemata, which are "latent but reactive" (Segal \& Shaw, 1986). Once activated negative schemata can bias self-related processing in a maladaptive way. In keeping with this, Marchetti et al. (2012) hypothesized that during rest increased TN activity could neurobiologically support such "latent but reactive" negative self-schemata. Although there is some overlap between brooding and cognitive reactivity, brooding is considered part of stable tendency to ruminate (Treynor et al., 2003) whereas cognitive reactivity is clearly defined in relation to the encounter of stress (Teasdale, 1988).

Related to the association between TN and cognitive reactivity, Marchetti et al. (2012) also proposed an inversed relation between mindfulness and TN. Mindfulness is a mindset characterized by weak connections between the self and negative thoughts and feelings (Segal, Williams, \& Teasdale, 2002), associative connections that are the very core of the construct of cognitive reactivity. Recent studies confirmed this notion, showing that mindfulness and cognitive reactivity are negatively correlated and that after a mindfulness 
Running head: REST, SELF-FOCUS AND NEGATIVE MOOD

training negative effects of cognitive reactivity decrease (e.g. Kuyken et al., 2010).

Moreover, high levels of dispositional mindfulness have been associated with decreased restrelated activation (Way, Creswell, Eisenberger, \& Lieberman, 2010). In sum, individuals with high level of mindfulness may, during rest, have a reduced likelihood for self-attitudes and negative feelings and thoughts to occur, which mirrors the process of cognitive reactivity in a protective manner.

In our study, we aimed to investigate depression-related cognitive processes that are subserved by the DMN through a behavioral resting state paradigm. It has been argued by Broyd et al. (2009) that DMN research is crucial in discerning the pathogenesis of psychiatric disorders and that the DMN may become valuable in differential diagnosis. Moreover, insight into maladaptive cognitive processes during rest can provide new opportunities for clinical interventions. We opted for a behavioral resting state approach as, compared to fMRI research, it has larger ecological validity. That is, fMRI scanner background noise impacts on cognitive control (Hommel, Fischer, Colzato, van den Wildenberg, \& Cellini, 2012), and suppresses TN activation in a nonlinear way (Gaab, Gabrieli, \& Glover, 2008), making any conclusion potentially confounded in a non-controllable fashion. To overcome these limitations, we used a validated behavioral paradigm which allows making inferences on the underlying neural activity (Vanhaudenhuyse et al., 2011). Specifically, we asked participants to rest for about 25 minutes, keep their eyes closed, and avoid any structured thinking. They were then pseudo-randomly probed during this resting state about the level of internal and external focus. Crucially, in this paradigm event-related higher levels of internal focus have been mirrored by higher activation of TN brain areas, such as medioprefrontal cortex and posterior cingulate cortex, while more externally-oriented focus has been related to increased activation at level of TP (i.e. inferior frontal gyrus and inferior parietal cortex). Moreover, internal and external focus showed the anticorrelated pattern typically occurring at the 
Running head: REST, SELF-FOCUS AND NEGATIVE MOOD

neurobiological level between TN and TP. Also the frequency of the switching internalexternal focus has been reported to be in the same range $(<0.1 \mathrm{~Hz})$ of DMN fluctuations (Vanhaudenhuyse et al., 2011). Compared with standard fRMI studies, our paradigm has the advantage to be noise-free and in turn immune from the biasing effect of constant auditory stimulation. The resting periods in between thought probes (mean $\sim 19 \mathrm{sec}$ ), were chosen to be sufficient to elicit consistent DMN activation (Gaab et al., 2008) and for participants to experience wandering thoughts (Flavell, Green, \& Flavell, 2000).

We hypothesized that during rest increased levels of internal focus would be the ideal condition for rumination-like analytical process to occur and, in turn, detrimentally impact on mood. In other words, given the tendency of internal focus towards self-related content, higher accessibility of self-salient information could facilitate comparative and judgmental processing (Teasdale, 1999), which elicits negative mood (Mor \& Winquist, 2002). In accord with our neurobiological framework (Marchetti et al., 2012), we expected that negative effects of internal focus on mood via contribution of ruminative self-focus would occur only in individuals with high levels of habitual brooding tendencies and/or cognitive reactivity. In keeping with this, we also predicted the absence of this effect in individuals who adopt a mindful perspective towards their thoughts. Moreover, we hypothesized that internal focus would only lead to lower mood when self-related information was processed in a ruminative way. Otherwise self-related internal focus was not expected per se to lower mood.

\section{Method}

\subsection{Participants}

Eighty-two undergraduates participated in this study. Two cases constituting an outlier (z point $>3$ ) were dropped, leaving 80 individuals (mean age $=20.5$ years, $S D=4.5,80 \%$ female). The study was approved by the local Ethical Committee. 


\subsection{Materials}

\section{$\underline{\text { Trait and state questionnaires }}$}

Depressive symptoms level was measured by the Beck Depression Inventory $2^{\text {nd }}$ Edition (BDI-II; Beck, Steer, \& Brown, 1996), whereas trait rumination was assessed by the Ruminative Responses Scale (RRS; Treynor et al., 2003). Then cognitive reactivity and mindfulness levels were measured respectively by the Leiden Index of Depression Sensitivity - Revised (LEIDS-R; Van der Does \& Williams, 2003) and the Mindful Attention Awareness Scale (MAAS; Brown \& Ryan, 2003).

Two state measures were acquired. Specifically, state mood was assessed by the Positive Affect Negative Affect Scale - State (PANAS - State; Watson, Clark, \& Tellegen, 1988), whereas state levels of ruminative self-focus were evaluated by the recently validated 6-item Momentary Ruminative Self-focus Items (MRSI; Mor, Marchetti, \& Koster, in preparation). Ruminative self-focus is defined as a temporary maladaptive analytical process, characterized by "thinking about" experience (Teasdale, 1999).

\section{$\underline{\text { Resting state paradigm }}$}

Attentional dynamics during rest were studied by means of a validated paradigm (Vanhaudenhuyse et al., 2011). At the start of the procedure, participants received the following instruction: "During the next 25 minutes, we ask you to keep your eyes closed and to avoid prolonged structured thinking, such as counting or singing. When you hear a beep, please use the keyboard to communicate the intensity of 'external awareness' and 'internal awareness' ongoing prior to the beep. 'External' is here defined as the perception of environmental sensory stimuli (e.g. auditory, visual, olfactory, or somesthetic). 'Internal' here refers to all environmental stimuli independent thoughts (e.g. inner speech, autobiographical 
Running head: REST, SELF-FOCUS AND NEGATIVE MOOD

memories, or wandering thoughts)". All participants were instructed to respond by using button presses from $1=$ absent to $7=$ maximal. After a training phase of 11 attention probes, the experiment phase consisted of 66 attention probes. Interstimulus interval was randomized between 11.3 and $26.8 \mathrm{sec}$.

This paradigm generates two main variables, "internal focus" (i.e. "internal awareness") and "external focus" (i.e. "external awareness"). For both internal and external focus, the mean scores across 66 attention probes were computed. Notably, in our study internal and external focus were significantly anticorrelated $(\mathrm{r}=-.42, p<.001)$.

\subsection{Design}

Participants filled in current mood state measures (PANAS-t0), followed by the state measure of ruminative self-focus (MRSI-t0). Then, individuals underwent the resting state phase, after which both measures of ruminative self-focus and mood were re-administered (respectively MRSI-t1 and PANAS-t1). Finally, questionnaires for individual differences in depressive symptoms, rumination, cognitive reactivity, and mindfulness (BDI-II, RRS, LEIDS-R, and MAAS) were administered.

\subsection{Data-analytic strategy}

We tested the hypothesis that higher levels of internal focus (focal predictor) could predict increased ruminative self-focus (intervening variable) after the experimental paradigm which in turn could explain higher levels of negative mood (outcome variable) (Figure 1B, C). Note that we had theoretical reasons to hypothesize neither total (path $c$, Figure 1A) nor direct (path c' Figure 1B, C) contribution of the focal predictor on the outcome variable. This type of statistical model is defined as indirect effect model (Mathieu \& Taylor, 2006). 
Running head: REST, SELF-FOCUS AND NEGATIVE MOOD

Additionally, we expected the indirect effect to vary across different levels of moderator(s), namely either cognitive reactivity and habitual brooding (Figure 1B) or mindfulness (Figure 1C). To test such conditional indirect effect, we performed all the analyses with Hayes'

(2012) PROCESS macro for SPSS (for more details see the Supplemental Online Material).

\section{Results}

Descriptive statistics, Pearson's correlations, and Cronbach's alphas are provided in Table 1.

First, we tested the significance of the indirect effect of internal focus (focal predictor) on increase of negative mood (outcome variable) through contribution of increased ruminative self-focus (intervening variable), across different levels of cognitive risk factors, namely cognitive reactivity and trait brooding (Figure 1B). In order to remove noise variance unrelated to the focal predictor, we included individual scores on dispositional mindfulness, reflection, depressive symptoms, and external focus during the task as covariates ${ }^{2}$.

Table 2 (upper part) shows that the investigated indirect effect is always significant (i.e. bootstrap CIs do not contain zero), with the only exception of low levels of cognitive reactivity. Moreover, inspection of the conditional indirect effect coefficients allows inferring that brooding has a smaller impact compared with cognitive reactivity; nevertheless given a certain level of cognitive reactivity, higher levels of brooding contribute to a stronger indirect path. Furthermore, the conditional effect of internal focus per either cognitive reactivity or brooding (path $a$ ) positively predicts increased ruminative self-focus (respectively $\beta=.28$ and $\beta=.05$ ), which in turn (path $b$ ) positively explains the increase of negative mood $(\beta=.24)$ (see Table S1 in Supplemental Material). On the contrary, the total effect of internal focus in predicting increased negative mood (Figure 1A, path $c$ ), after controlling for the variables 
Running head: REST, SELF-FOCUS AND NEGATIVE MOOD

implicated in the previous model, is not significant (see lower part Table S1); neither is the direct effect (Figure 1B, path $c^{\prime}$ ) (middle part Table S1). To summarize, higher levels of internal focus during rest predict increased levels of negative mood via contribution of increased ruminative self-focus. This condition holds only for moderate and high levels of cognitive reactivity, whereas the contribution of brooding, although still in line with our hypothesis, is smaller.

Second, we tested the significance of the indirect effect of internal focus (focal predictor) on the increase of negative mood (outcome variable) through contribution of increased ruminative self-focus (intervening variable), across different levels of mindfulness as protective factor (Figure 1C). Here we included the following covariates: external focus, depressive symptoms, brooding, reflection, and cognitive reactivity. Table 2 (lower part) shows that the investigated indirect effect is always significant, with the exception of high levels of mindfulness. As hypothesized, the conditional effect of internal focus moderated by mindfulness (path $a$ ) negatively predicts increased ruminative self-focus $(\beta=-.18$ ), which in turns (path $b$ ) positively explains the increase of negative mood $(\beta=.30)$ (see Table S3). On the contrary, neither the total effect (path $c$ ) nor the direct effect (path $c^{\prime}$ ) of internal focus significantly predicted increased negative mood, after controlling for variables included in the conditional indirect effect model (see middle and lower part Table S3). To summarize, higher levels of internal focus during rest positively predict increased levels of negative mood via contribution of increased ruminative self-focus. This condition does not hold for individuals showing high levels of dispositional mindfulness.

\section{Discussion}

Rest is increasingly being the focus of intense research and several neurobiological abnormalities have been reported during resting state in psychopathology (Whitfield-Gabrieli 
\& Ford, 2012). Resting state provides an interesting condition to study depressive cognitive risk factors and protective factors (Marchetti et al., 2012) as behavioral and neuropsychological studies have associated rest with heightened levels of self-related processing (Northoff, 2012). Nevertheless, little is known about depressogenic cognitive processes during rest. Therefore, we adopted a behavioral noise-free paradigm to study cognitive processes that influence mood during resting state. The main finding of our study is that there is an indirect effect of internal focus on negative mood through ruminative selffocus when individuals have moderate to high cognitive reactivity, whereas brooding seems to have only an incremental effect. This indirect path is not significant in individuals with low cognitive reactivity (regardless of brooding levels), or high mindfulness levels.

The significant indirect path indicates that internally focused attention is a condition that can facilitate judgmental and analytical thinking, which subsequently heightens negative mood. This is in line with theories relating self-focused attention to negative mood and depression (Ingram, 1990). It is noteworthy that internal focus during rest did not overlap with state ruminative self-focus, as these two variables were only moderately correlated.

Crucially, our data strongly indicates that there are individual differences in the affective consequences of being internally focused during rest (i.e. conditional indirect effect). On the one hand, cognitive reactivity levels strongly determined the strength by which internal focus predicted worsening in mood via ruminative self-focus. On the other hand, brooding appears to have only an incremental effect in that, given a certain level of cognitive reactivity, brooding augmented the indirect effect without actually influencing individuals with low levels of cognitive reactivity. It is noteworthy that we have been able to specify the contribution of cognitive reactivity above and beyond maladaptive rumination, as shown in previous research (Moulds et al., 2008). Although being highly correlated ( $r=.60)$, these two 
constructs show a hierarchical asymmetry in moderating the indirect effect, with cognitive reactivity dominating over brooding.

Our study also shows individual difference variables that have a protective effect in such contexts. Individuals with high levels of mindfulness did not experience a worsening in mood. The construct of mindfulness is thought to represent weakened links between the self, (negative) thinking, and (negative) feelings (Way et al., 2010), and in turn to conceptually oppose cognitive reactivity (Marchetti et al., 2012, Raes et al., 2009). Correlational analysis confirms this hypothesis, showing that mindfulness negatively correlates only with cognitive reactivity, but not with brooding ${ }^{3}$. A possible explanation for this result is that, despite the emergence of self-related material during rest, mindful individuals are capable of not engaging in a self-judgmental loop.

Why is this study of resting state of theoretical and clinical importance? Typically during rest when there is time for self-reflection, individuals experience the most negative thoughts. However, such conditions have so far rarely been investigated in lab contexts. The current paradigm used is highly promising to further study rest-related phenomena in psychopathology, with a strong link to rapidly advancing DMN research (Whitfield-Gabrieli \& Ford, 2012). Although the current paradigm is less informative about the neural mechanisms, it is noteworthy that resting state as studied through this behavioral paradigm has a larger ecological validity than studies using fMRI. By capitalizing on this, we aimed to bridge the extant gap between clinical theories and basic neuroscience. Second, our findings provide evidence for interplay between cognitive risk as well as protective factors. In line with a recent proposal (Marchetti et al., 2012), we could relate different aspects of depression risk to resting state and DMN. Especially observing these relations in a sample of healthy, non-depressed participants suggests that there are strong relations between resting state, negative thinking, and negative mood which can turn toxic even in individuals with limited 
Running head: REST, SELF-FOCUS AND NEGATIVE MOOD

levels of psychopathology. Therefore, it is conceivable that clinically depressed individuals during rest might show stronger effects. Our findings can also account for the clinical observation that task engagement can lift negative mood (Hopko, Lejuez, Ruggiero, \& Eifert, 2003). Finally, in relation to clinical interventions, mindfulness has promising effects in decreasing cognitive risk factors (Ma \& Teasdale, 2004). Given the high frequency of (short) resting state phases in everyday life, it appears important for at-risk individuals (e.g., remitted depressed individuals) to develop mindful skills which can protect them from negative consequences associated with rest.

Our study has limitations. First, despite a strong link has been established between internal/external focus and the DMN activity during rest (Vanhaudenhuyse et al., 2011), in our study we can only indirectly relate our results to activity of underlying neural mechanisms. Second, our study is correlational in nature so no conclusions can be drawn regarding causal relationships. Therefore, future studies should further investigate the reported effects by directly manipulating the degree of internal focus during rest. Third, the presence of probes during the task might have influenced the investigated phenomenon. However, previous research on mindwandering has shown that assessment of mindwandering using thought probes generates similar results as and, sometimes, better than retrospective reports (Hurlburt \& Melancon, 1987).

To conclude, we found that internal focus has a detrimental effect on negative mood through ruminative self-focus only when individuals have moderate to high cognitive reactivity, whereas this is not the case in low cognitive reactivity or high levels of mindfulness. Resting state clearly is a pivotal moment where negative cognitions can creep up on individuals. Our study attempted to bridge the literature on cognitive and neural mechanisms involved in resting state, which increases our understanding of risk as well as protective influences. 
Running head: REST, SELF-FOCUS AND NEGATIVE MOOD

\section{Acknowledgements}

This research was supported by a Grant of the Special Research Fund (BOF) of Ghent University (BOF 10/2JO/061) awarded to Ernst Koster and a Grant BOF10/GOA/014 for a Concerted Research Action of Ghent University awarded to Rudi De Raedt and Ernst Koster. 
Running head: REST, SELF-FOCUS AND NEGATIVE MOOD

\section{References}

Beck, A.T., Steer, R.A., \& Brown, G.K. (1996). Manual for the Beck Depression Inventory-II. San Antonio, TX: Psychological Corporation.

Berman, M. G., Peltier, S., Nee, D. E., Kross, E., Deldin, P. J., \& Jonides, J. (2011). Depression, rumination and the default network. Social Cognitive and Affective Neuroscience, 6(5), 548-555.

Brown, K. W., \& Ryan, R. M. (2003). The benefits of being present: Mindfulness and its role in psychological well-being. Journal of Personality and Social Psychology, 84(4), $822-848$.

Broyd, S. J., Demanuele, C., Debener, S., Helps, S. K., James, C. J., \& Sonuga-Barke, E. J. S. (2009). Default-mode brain dysfunction in mental disorders: A systematic review. Neuroscience and Biobehavioral Reviews, 33(3), 279-296.

Buckner, R. L., Andrews-Hanna, J. R., \& Schacter, D. L. (2008). The brain's default network - Anatomy, function, and relevance to disease. Annals of the New York Academy of Sciences 1124, 1-38.

Butler, A. C., Hokanson, J. E., \& Flynn, H. A. (1994). A Comparison of Self-Esteem Lability and Low Trait Self-Esteem as Vulnerability Factors for Depression. Journal of Personality and Social Psychology, 66(1), 166-177.

Corbetta, M., \& Shulman, G. L. (2002). Control of goal-directed and stimulus-driven attention in the brain. Nature Reviews Neuroscience, 3(3), 201-215.

Flavell, J. H., Green, F. L., \& Flavell, E. R. (2000). Development of Children's Awareness of Their Own Thoughts. Journal of Cognition and Development, 1(1), 97-112.

Fox, M. D., Snyder, A. Z., Vincent, J. L., Corbetta, M., Van Essen, D. C., \& Raichle, M. E. (2005). The human brain is intrinsically organized into dynamic, anticorrelated 
Running head: REST, SELF-FOCUS AND NEGATIVE MOOD

functional networks. Proceedings of the National Academy of Sciences of the United States of America, 102(27), 9673-9678.

Gaab, N., Gabrieli, J. D. E., \& Glover, G. H. (2008). Resting in peace or noise: Scanner background noise suppresses default-mode network. Human Brain Mapping, 29(7), $858-867$.

Hamilton, J. P., Furman, D. J., Chang, C., Thomason, M. E., Dennis, E., \& Gotlib, I. H. (2011). Default-Mode and Task-Positive Network Activity in Major Depressive Disorder: Implications for Adaptive and Maladaptive Rumination. Biological Psychiatry, 70(4), 327-333.

Hayes, A. F. (2012). An Analytical Primer and Computational Tool for observed Variable Mediation, Moderation, and Conditional Process Modeling. Manuscript submitted for publication.

Hommel, B., Fischer, R., Colzato, L. S., van den Wildenberg, W. P. M., \& Cellini, C. (2012). The Effect of fMRI (Noise) on Cognitive Control. Journal of Experimental Psychology-Human Perception and Performance, 38(2), 290-301.

Hopko, D. R., Lejuez, C. W., Ruggiero, K. J., \& Eifert, G. H. (2003). Contemporary behavioral activation treatments for depression: Procedures, principles, and progress. Clinical Psychology Review, 23(5), 699-717.

Hurlburt, R. T., \& Melancon, S. M. (1987). How Are Questionnaire Data Similar to, and Different from, Thought-Sampling Data - 5 Studies Manipulating Retrospectiveness, Single-Moment Focus, and Indeterminacy. Cognitive Therapy and Research, 11(6), 681-703.

Ingram, R. E. (1990). Self-Focused Attention in Clinical Disorders - Review and a Conceptual-Model. Psychological Bulletin, 107(2), 156-176. 
Running head: REST, SELF-FOCUS AND NEGATIVE MOOD

Kuyken, W., Watkins, E., Holden, E., White, K., Taylor, R. S., Byford, S., . . Dalgleish, T. (2010). How does mindfulness-based cognitive therapy work? Behaviour Research and Therapy, 48(11), 1105-1112.

Ma, S. H., \& Teasdale, J. D. (2004). Mindfulness-based cognitive therapy for depression: Replication and exploration of differential relapse prevention effects. Journal of Consulting and Clinical Psychology, 72(1), 31-40.

Marchetti, I., Koster, E. H., Sonuga-Barke, E. J., \& De Raedt, R. (2012). The Default Mode Network and recurrent depression: A neurobiological model of cognitive risk factors. Neuropsychology Review, 22(3), 229-251.

Mathieu, J. E., \& Taylor, S. R. (2006). Clarifying conditions and decision points for mediational type inferences in organizational behavior. Journal of Organizational Behavior, 27(8), 1031-1056.

Mor, N., \& Winquist, J. (2002). Self-focused attention and negative affect: A meta-analysis. Psychological Bulletin, 128(4), 638-662.

Moulds, M. L., Kandris, E., Williams, A. D., Lang, T., Yap, C., \& Hoffmeister, K. (2008). An investigation of the relationship between cognitive reactivity and rumination. Behavior Therapy, 39(1), 65-71.

Nolen-Hoeksema, S. (1991). Responses to Depression and Their Effects on the Duration of Depressive Episodes. Journal of Abnormal Psychology, 100(4), 569-582.

Northoff, G. (2012). Immanuel Kant's mind and the brain's resting state. Trends in Cognitive Sciences, 16(7), 356-359.

Raichle, M. E., MacLeod, A. M., Snyder, A. Z., Powers, W. J., Gusnard, D. A., \& Shulman, G. L. (2001). A default mode of brain function. Proceedings of the National Academy of Sciences of the United States of America, 98(2), 676-682. 
Rude, S. S., Maestas, K. L., \& Neff, K. (2007). Paying attention to distress: What's wrong with rumination? Cognition \& Emotion, 21(4), 843-864.

Segal, Z. V., \& Shaw, B. F. (1986). Cognition in Depression - a Reappraisal of Coyne and Gotlib Critique. Cognitive Therapy and Research, 10(6), 671-693.

Segal, Z. V., Williams, J. M., \& Teasdale, J. (2002). Mindfulness-based cognitive therapy for depression: A new approach to preventing relapse. New York: Guilford Press.

Teasdale, J. D. (1988). Cognitive vulnerability to persistent depression. Cognition \& Emotion, 2, 247-274.

Teasdale, J. D. (1999). Emotional processing, three modes of mind and the prevention of relapse in depression. Behaviour Research and Therapy, 37, S53-S77.

Treynor, W., Gonzalez, R., \& Nolen-Hoeksema, S. (2003). Rumination reconsidered: A psychometric analysis. Cognitive Therapy and Research, 27(3), 247-259.

Van der Does, A., \& Williams, J. M. (2003). Leiden Index of Depression Sensitivity Revised (LEIDS-R). Retrieved 10th February 2012, from http://www.dousa.nl/publications_depression.htm\#LEIDS

Vanhaudenhuyse, A., Demertzi, A., Schabus, M., Noirhomme, Q., Bredart, S., Boly, M., .. . Laureys, S. (2011). Two Distinct Neuronal Networks Mediate the Awareness of Environment and of Self. Journal of Cognitive Neuroscience, 23(3), 570-578.

Watson, D., Clark, L. A., \& Tellegen, A. (1988). Development and Validation of Brief Measures of Positive and Negative Affect - the Panas Scales. Journal of Personality and Social Psychology, 54(6), 1063-1070.

Way, B. M., Creswell, J. D., Eisenberger, N. I., \& Lieberman, M. D. (2010). Dispositional Mindfulness and Depressive Symptomatology: Correlations With Limbic and SelfReferential Neural Activity During Rest. Emotion, 10(1), 12-24. 
Running head: REST, SELF-FOCUS AND NEGATIVE MOOD

Whitfield-Gabrieli, S., \& Ford, J. M. (2012). Default Mode Network Activity and Connectivity in Psychopathology. Annual Review of Clinical Psychology, 8, 49-76. 
Running head: REST, SELF-FOCUS AND NEGATIVE MOOD

\section{Footnotes}

${ }^{1}$ : Impaired attentional control will not be considered here as it is proposed to specifically occur in the transition between rest and task.

${ }^{2}$ : Mindfulness is considered to negatively mirror cognitive reactivity as it also emerged in the correlational analysis $(\mathrm{r}=-.42)$. By controlling for mindfulness, we were able to estimate the particular effect of cognitive reactivity above and beyond mindfulness. For the same reason, we included reflection. Despite their positive relation, reflection and brooding have been reported to show an opposite relationship in relation to the $\mathrm{TN}$ at neurobiological level (Berman et al., 2011; Hamilton et al., 2011). This observation was confirmed by the opposite role of both reflection and brooding in predicting the increase of ruminative self-focus (see upper part Table S1). Moreover, individual level of dysphoria was included given its wellestablished contribution to rumination and negative mood. Importantly, none of the covariates, except for external focus ( $\mathrm{r}=-.42)$, was significantly correlated with the focal predictor, a condition which makes such statistical controlling fully appropriate (Miller \& Chapman, 2001). External focus was included as covariate to ensure that findings obtained for internal focus were not due to dynamics related to external focus of attention.

${ }^{3}$ : This pattern holds even after controlling for brooding $\left(\mathrm{r}_{\mathrm{p}}=-.382, p<.001\right)$, on the contrary the relation between brooding and mindfulness is reduced to zero after controlling for cognitive reactivity $\left(\mathrm{r}_{\mathrm{p}}=.07, p=\mathrm{ns}\right)$. 
Running head: REST, SELF-FOCUS AND NEGATIVE MOOD

\section{Figure Captions}

Figure 1: Hypothesized conditional indirect effect models. 1A represents the total effect of internal focus in predicting increased negative mood (path $c$ ). 1B represents the model for cognitive risk factors, that is both the direct effect (path $c^{\prime}$ ) and conditional indirect effect per different levels cognitive reactivity and brooding (path $a x b$ ) are depicted. 1C represents the model for protective factor, that is both the direct effect (path $c^{\prime}$ ) and conditional indirect effect per different levels of mindfulness (path $a x b$ ) are shown. 
Table 1. Mean, standard deviation and Cronbach's alpha $(n=80)$

\begin{tabular}{|c|c|c|c|c|c|c|c|c|c|c|c|c|c|c|c|}
\hline & $M$ & $S D$ & (2) & (3) & (4) & (5) & (6) & (7) & (8) & (9) & (10) & (11) & (12) & (13) & (14) \\
\hline (1) Internal Focus & 4.86 & 0.93 & $-.42 * * *$ & $.46 * * *$ & .03 & $.36 * * *$ & .11 & -.07 & .02 & -.13 & .01 & -.13 & .02 & -.10 & $\begin{array}{l}.01 \\
\end{array}$ \\
\hline (2) External Focus & 2.88 & 0.84 & - & -.09 & .08 & .00 & .05 & .18 & $.23^{*}$ & .18 & .06 & .16 & .02 & .01 & -.03 \\
\hline (3) $\Delta$ MRSI t1-t0 & 2.30 & 4.85 & & - & -.15 & $.59 * * *$ & $.25^{*}$ & -.01 & .19 & -.05 & .06 & -.18 & .17 & .00 & .05 \\
\hline (4) MRSI-t0 & 23.31 & 5.54 & & & $(.68)$ & $.71^{* * *}$ & -.09 & $.30 * *$ & $.23^{*}$ & $.34 * * *$ & $.34 * * *$ & $.22 *$ & $.35^{* * *}$ & $.26 * *$ & -.04 \\
\hline (5) MRSI-t1 & 25.61 & 6.80 & & & & $(.79)$ & .10 & $.24^{*}$ & $.33 * * *$ & $.25^{*}$ & $.32 *$ & .05 & $.41^{* * *}$ & .21 & .00 \\
\hline (6) $\triangle$ PANAS negative t1-t0 & -1.05 & 3.23 & & & & & - & $-.43 * * *$ & $.36 * * *$ & -.11 & -.07 & -.05 & -.15 & -.21 & .07 \\
\hline (7) PANAS negative-t0 & 14.28 & 4.14 & & & & & & $(.80)$ & $.69 * * *$ & $.51^{* * *}$ & .20 & .06 & $.31 * *$ & .17 & -.07 \\
\hline (8) PANAS negative-t1 & 13.23 & 4.02 & & & & & & & $(.81)$ & $.43^{* * *}$ & .15 & .02 & .19 & .01 & -.02 \\
\hline (9) BDI-II & 9.41 & 6.53 & & & & & & & & $(.87)$ & $.31 *$ & .20 & $.28 * *$ & $.27 * *$ & -.16 \\
\hline (10) RRS Total & 45.27 & 12.23 & & & & & & & & & $(.91)$ & $.69 * * *$ & $.80^{* * *}$ & $.66^{* * *}$ & -.13 \\
\hline (11) RRS Reflection & 10.18 & 3.81 & & & & & & & & & & $(.82)$ & $.32 * *$ & $.38 * * *$ & .00 \\
\hline (12) RRS Brooding & 10.45 & 3.50 & & & & & & & & & & & $(.78)$ & $.60 * * *$ & -.20 \\
\hline (13) LEIDS-R & 42.61 & 16.54 & & & & & & & & & & & & $(.88)$ & $-.42 * * *$ \\
\hline (14) MAAS & 3.75 & 0.61 & & & & & & & & & & & & & $(.78)$ \\
\hline
\end{tabular}

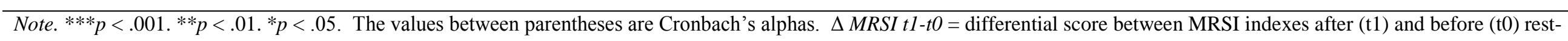

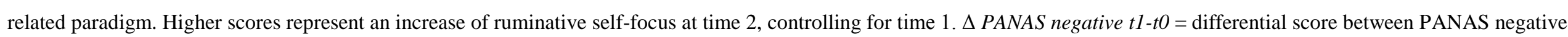
indexes after (t1) and before (t0) rest-related paradigm. Higher scores represent an increase of negative mood at time 2, controlling for time 1. 
Table 2. Conditional indirect effect of internal focus on the increase of negative mood across low (-1 SD), medium (M) and high (+1) levels of cognitive reactivity and brooding via increased ruminative self-focus (upper part); conditional indirect effect across low (-1 $S D)$, medium $(M)$ and high $(+1)$ levels of mindfulness (lower part) $(n=80)$

\begin{tabular}{clcccc}
\hline $\begin{array}{c}\text { Cognitive } \\
\text { reactivity }\end{array}$ & Brooding & $\begin{array}{c}\text { Conditional } \\
\text { indirect effect }\end{array}$ & Boot SE & $\begin{array}{c}\text { Boot LL CI } \\
95 \%\end{array}$ & $\begin{array}{c}\text { Boot UL CI } \\
95 \%\end{array}$ \\
\hline \multirow{3}{*}{ Low } & Low & .16 & .15 & -.02 & .65 \\
& Medium & .21 & .16 & -.02 & .66 \\
& High & .26 & .28 & -.11 & 1.11 \\
\hline \multirow{3}{*}{ Medium } & Low & .39 & .29 & .01 & 1.15 \\
& Medium & .44 & .24 & .01 & .96 \\
& High & .49 & .29 & .03 & 1.20 \\
\hline \multirow{3}{*}{ High } & Low & .62 & .45 & .03 & 1.91 \\
& Medium & .66 & .39 & .01 & 1.58 \\
& High & .71 & .39 & .01 & 1.55 \\
\hline \hline \multirow{2}{*}{ Mindfulness } & & Conditional & Boot SE & Boot LL CI & Boot UL CI \\
& & indirect effect & & $.36 \%$ & $95 \%$ \\
\hline \multirow{2}{*}{} & Low & .74 & .36 & .13 & 1.55 \\
& Medium & .49 & .23 & .09 & 1.02 \\
& High & .25 & .23 & -.06 & .92 \\
\hline
\end{tabular}




\section{Supplemental Online Material}

\section{Data-analytic strategy}

In our study, we aimed at testing a specific statistical model where only the indirect effect $(a x b)$ is expected to be significant, neither the total (c) nor the direct effect ( $\left.c^{\prime}\right)$ is expected to be significant. Indirect effect model is a term which has been proposed to differentiate from full and partial meditational models where significant either total or direct effects are expected (Preacher \& Hayes, 2008). In keeping with the guidelines proposed by Mathieu and Taylor (2006), we first tested the statistical significance of the indirect effect, operationalized as the product of path $a$ and $b$. We then tested the null total effect of the focal predictor on the outcome variable without taking into account the contribution of the intervening variable (Figure 1A). We also tested the null effect of the direct path (c'), namely the contribution of the focal predictor on the outcome after controlling for the intervening variable. When these three conditions were satisfied, we could test whether the relation between the focal predictor and the outcome is due to an indirect effect through the contribution of the intervening variable (Hayes, 2009; Hayes, Preacher, \& Myers, 2011; Mathieu \& Taylor, 2006). Nevertheless, the test of the indirect effect model was performed on data that were only partially structured to be temporally consistent with the proposed underlying process, with the intervening variable (increased ruminative self-focus) and the outcome variable (increased negative mood) being measured at the same time. In order to control for the alternative indirect path (reversed model), the data were subjected to an analysis in which the increased negative mood served as intervening variable and the enhanced ruminative self-focus as outcome. If the results of this reversed model were also significant, caution would be warranted (Kenny, 2012). 
To test the statistical significance of the indirect effect (path $a x b$ ), according to Preacher and Hayes' recommendations (2008) we adopted the nonparametric bootstrapping approach. Compared with the causal steps approach (Baron \& Kenny, 1986) or the Sobel test (Sobel, 1982), bootstrapping is considered the most powerful approach and free from unrealistic assumptions, such as the multivariate normality in data distribution (Bollen \& Stine, 1990; Hayes, 2009). Specifically, bootstrapping circumvents this problem by relying on confidence intervals (CIs) for the determination of the indirect effect instead of the traditional p-value approach that uses standard errors (MacKinnon, Lockwood, \& Williams, 2002). According to Preacher and Hayes (2008), to test the significance of the indirect effect (path $a x b$ ) we estimated 10000 bootstrap bias-corrected 95\% CIs, and if they did not contain zero they were considered significant. Crucially, to determine the significance of the indirect effect ( $a x b$ path) we evaluated only the bootstrap CIs without considering the significance of path $a$ and $b$, as recommended by Hayes (2009, 2012). Regressions weights for both path $a$ and $b$ were computed only to clarify the direction of the influence of the focal predictor over the intervening variable (path $a$ ) and the intervening variable over the outcome variable (path $b$ ).

Furthermore, we put forward the indirect effect to vary across different levels of the moderator(s), either cognitive reactivity and brooding (Figure 1B) or mindfulness (Figure 1C). For both models, we estimated different conditional indirect effects of the focal predictor over the outcome variable at low (one SD below the mean), moderate (sample mean), and high (one SD above the mean) values of the moderator(s). Following Cohen, Cohen, West, and Aiken (2003), both the focal predictor and the moderator(s) were mean-centered prior calculating the interaction term(s).

\section{Additional Analyses}


We tested the reversed models, where increased negative mood acts as intervening variable and increased ruminative self-focus acts as outcome variable, by estimating the indirect effect. Specifically, we ruled out the statistical significance of the reversed models for both cognitive risk factors (TableS2, upper part) and protective factor (TableS2, lower part).

\section{References}

Baron, R. M., \& Kenny, D. A. (1986). The Moderator Mediator Variable Distinction in Social Psychological-Research - Conceptual, Strategic, and Statistical Considerations. Journal of Personality and Social Psychology, 51(6), 1173-1182.

Bollen, K. A., \& Stine, R. (1990). Direct and indirect effects: Classical and bootstrap estimates of variability. Sociological Methodology, 20, 115-140.

Cohen, J., Cohen, P., West, S. G., \& Aiken, L. S. (2003). Applied Multiple Regression/Correlation Analysis for the Behavioral Sciences (3rd Edition). Mahwah, NJ.: Erlbaum.

Hayes, A. F. (2009). Beyond Baron and Kenny: Statistical Mediation Analysis in the New Millennium. Communication Monographs, 76(4), 408-420.

Hayes, A. F. (2012). An Analytical Primer and Computational Tool for observed Variable Mediation, Moderation, and Conditional Process Modeling. Manuscript submitted for publication.

Hayes, A. F., Preacher, K. J., \& Myers, L. J. (2011). Mediation and the estimation of indirect effects in political communication research. In E. P. Bucy \& R. Lance Holbert (Eds.), Sourcebook for political communication research: Methods, measures, and analytical techniques (pp. 434-465). New York: Routledge. 
Kenny, D. (2012). Mediation: Specification error. Retrieved 3rd April 2012, from http://davidakenny.net/cm/mediate.htm\#SE

MacKinnon, D. P., Lockwood, C. M., Hoffman, J. M., West, S. G., \& Sheets, V. (2002). A comparison of methods to test mediation and other intervening variable effects. Psychological Methods, 7(1), 83-104.

Mathieu, J. E., \& Taylor, S. R. (2006). Clarifying conditions and decision points for mediational type inferences in organizational behavior. Journal of Organizational Behavior, 27(8), 1031-1056.

Preacher, K. J., \& Hayes, A. F. (2008). Asymptotic and resampling strategies for assessing and comparing indirect effects in multiple mediator models. Behavior Research Methods, 40(3), 879-891.

Sobel, M. E. (1982). Asymptotic confidence intervals for indirect effects in structural equation models. In S. Leinhardt (Ed.), Sociological methodology 1982 (pp. 290-312). Washington, DC: American Sociological Association. 
Table S1. Summary regressions for the total and direct effect of internal focus over increased negative mood conditioned to cognitive reactivity and brooding via increased ruminative self-focus $(n=80)$

\begin{tabular}{|c|c|c|c|c|c|}
\hline \multirow[t]{10}{*}{$\Delta$ MRSI t1-t0 } & Predictor & $B$ & $S E B$ & $\beta$ & $\overline{\mathrm{R}^{2}}$ \\
\hline & BDI-II & -.01 & .07 & -.01 & \\
\hline & Reflection & -.37 & 13 & $-.29 * *$ & \\
\hline & Brooding & .35 & .16 & $.25 *$ & \\
\hline & LEIDS-R & .03 & .04 & .09 & \\
\hline & MAAS & 1.59 & .86 & .20 & \\
\hline & External focus & 1.01 & .62 & 17 & \\
\hline & Internal focus & 2.76 & .54 & $.53^{* * *}$ & \\
\hline & Internal focus x LEIDS-R (a) & .08 & .04 & $.28^{*}$ & \\
\hline & Internal focus x Brooding $(a)$ & .08 & .21 & .05 & $.401^{* * *}$ \\
\hline \multirow{7}{*}{$\begin{array}{l}\text { PANAS } \\
\text { negative t1-t0 }\end{array}$} & Predictor & $B$ & $S E B$ & $\beta$ & $\mathrm{R}^{2}$ \\
\hline & BDI-II & -.05 & .06 & -.11 & \\
\hline & Reflection & .00 & .10 & .00 & \\
\hline & MAAS & .22 & .60 & .04 & \\
\hline & External focus & .44 & .48 & .11 & \\
\hline & Internal focus ( $\left.c^{\prime}\right)$ & .13 & .48 & .04 & \\
\hline & $\Delta$ MRSI t1-t0 $(b)$ & .16 & .08 & .24 & .086 \\
\hline \multirow{8}{*}{$\begin{array}{l}\Delta \text { PANAS } \\
\text { negative t1-t0 }\end{array}$} & Predictor & $B$ & $S E B$ & $\beta$ & $\mathrm{R}^{2}$ \\
\hline & BDI-II & -.03 & .06 & -.06 & \\
\hline & Reflection & .04 & .11 & .05 & \\
\hline & Brooding & -.05 & .13 & -.06 & \\
\hline & LEIDS-R & -.03 & .03 & -.17 & \\
\hline & MAAS & -.12 & .68 & -.02 & \\
\hline & External focus & .48 & .49 & .13 & \\
\hline & Internal focus $(c)$ & .52 & .44 & 15 & .073 \\
\hline
\end{tabular}

Note. ${ }^{* * *} p<.001 ;{ }^{* *} p<.01 ;{ }^{*} p<.05 . \Delta$ MRSI $11-t 0=$ differential score between MRSI indexes after (t1) and before (t0) rest-related paradigm. Higher scores represent an increase of ruminative self-focus at time 2, controlling for time 1. $\triangle$ PANAS negative $t 1-t 0=$ differential score between PANAS negative indexes after $(\mathrm{t} 1)$ and before $(\mathrm{t} 0)$ rest-related paradigm. Higher scores represent an increase of negative mood at time 2, controlling for time 1. 
Table S2. Conditional indirect effect of internal focus on the increase of ruminative selffocus across low (-1 SD), medium (M) and high (+1)levels of cognitive reactivity and brooding via increased negative mood (reversed model) (upper part); conditional indirect effect across low (-1 SD), medium (M) and high (+1) levels of mindfulness (reversed model) (lower part) $(n=80)$

\begin{tabular}{clcccc}
\hline \hline $\begin{array}{c}\text { Cognitive } \\
\text { reactivity }\end{array}$ & Brooding & $\begin{array}{c}\text { Conditional } \\
\text { indirect effect }\end{array}$ & Boot SE & $\begin{array}{c}\text { Boot LL CI } \\
95 \%\end{array}$ & $\begin{array}{c}\text { Boot UL CI } \\
95 \%\end{array}$ \\
\hline \multirow{3}{*}{ Low } & Low & -.03 & .19 & -.51 & .31 \\
& Medium & .11 & .23 & -.21 & .78 \\
& High & .26 & .37 & -.24 & 1.37 \\
\hline \multirow{3}{*}{ Medium } & Low & .01 & .21 & -.34 & .53 \\
& Medium & .16 & .18 & -.03 & .72 \\
& High & .30 & .30 & -.06 & 1.18 \\
\hline \multirow{3}{*}{ High } & Low & 05 & .31 & -.43 & .92 \\
& Medium & .20 & .24 & -.05 & .97 \\
& High & .34 & .31 & -.02 & 1.20 \\
\hline \hline Mindfulness & & Conditional & Boot SE & Boot LL CI & Boot UL CI \\
& indirect effect & .27 & .26 & -.05 & $.95 \%$ \\
\hline & High & .16 & .19 & -.08 & .74 \\
& Medium & .06 & .26 & -.42 & .68 \\
\hline \hline
\end{tabular}


Table S3. Summary regressions for the total and direct effect of internal focus over increased negative mood conditioned to mindfulness via increased ruminative self-focus ( $n$ $=80$ )

\begin{tabular}{|c|c|c|c|c|c|}
\hline \multirow[t]{9}{*}{$\Delta$ MRSI t1-t0 } & Predictor & $B$ & SE B & $\bar{\beta} \beta$ & $\mathrm{R}^{2}$ \\
\hline & BDI-II & -.00 & .08 & -.01 & \\
\hline & Reflection & -.28 & .14 & $-.22 *$ & \\
\hline & Brooding & .26 & .17 & .19 & \\
\hline & LEIDS-R & .01 & .04 & .05 & \\
\hline & MAAS & 1.30 & .89 & .16 & \\
\hline & External focus & .88 & .63 & .15 & \\
\hline & Internal focus & 2.52 & .57 & $.48 * * *$ & \\
\hline & Internal focus x MAAS $(a)$ & -2.00 & 1.15 & -.18 & $.335 * * *$ \\
\hline \multirow{8}{*}{$\begin{array}{l}\triangle \mathrm{PANAS} \\
\text { negative t1-t0 }\end{array}$} & Predictor & $B$ & $S E B$ & $\beta$ & $\mathrm{R}^{2}$ \\
\hline & BDI-II & -.02 & .06 & -.05 & \\
\hline & Reflection & .09 & .10 & .11 & \\
\hline & Brooding & -.12 & .13 & -.13 & \\
\hline & LEIDS-R & -.03 & .03 & -.16 & \\
\hline & External focus & .32 & .48 & .08 & \\
\hline & Internal focus (c’) & .02 & .48 & .00 & \\
\hline & $\Delta$ MRSI t1-t0 $(b)$ & .20 & .09 & $.30 *$ & .135 \\
\hline \multirow[t]{8}{*}{$\begin{array}{l}\triangle \mathrm{PANAS} \\
\text { negative } \mathrm{t} 1-\mathrm{t} 0\end{array}$} & Predictor & $B$ & $S E B$ & $\beta$ & $\mathrm{R}^{2}$ \\
\hline & BDI-II & -.03 & .06 & -.06 & \\
\hline & Reflection & .04 & .11 & .05 & \\
\hline & Brooding & -.05 & .13 & -.06 & \\
\hline & LEIDS-R & -.03 & .03 & -.17 & \\
\hline & MAAS & -.12 & .68 & -.02 & \\
\hline & External focus & .48 & .49 & .13 & \\
\hline & Internal focus (c) & .52 & .44 & .15 & .073 \\
\hline
\end{tabular}

Note. ${ }^{* * *} p<.001 ;{ }^{* *} p<.01 ;{ }^{*} p<.05 . \Delta$ MRSI $t 1-t 0=$ differential score between MRSI indexes after (t1) and before (t0) rest-related paradigm. Higher scores represent an increase of ruminative self-focus at time 2 , controlling for time 1. $\triangle$ PANAS negative $t 1-t 0=$ differential score between PANAS negative indexes after $(\mathrm{t} 1)$ and before $(\mathrm{t} 0)$ rest-related paradigm. Higher scores represent an increase of negative mood at time 2 , controlling for time 1. 